\title{
Improving Underwater Visibility Using Vignetting Correction
}

\author{
K. Sooknanan ${ }^{a}$, A. Kokaram ${ }^{a}$, D. Corrigan ${ }^{a}$, G. Baugh ${ }^{a}$, J. Wilson ${ }^{b}$ and N. Harte ${ }^{a}$ \\ ${ }^{a}$ Department of Electrical Engineering, Trinity College Dublin, Dublin 2, Ireland. \\ ${ }^{b}$ Department of Zoology, Trinity College Dublin, Dublin 2, Ireland. *
}

\begin{abstract}
Underwater survey videos of the seafloor are usually plagued with heavy vignetting (radial falloff) outside of the light source beam footprint on the seabed. In this paper we propose a novel multi-frame approach for removing this vignetting phenomenon which involves estimating the light source footprint on the seafloor, and the parameters for our proposed vignetting model. This estimation is accomplished in a bayesian framework with an iterative SVD-based optimization. Within the footprint, we leave the image contents as is, whereas outside this region, we perform vignetting correction. Our approach does not require images with different exposure values or recovery of the camera response function, and is entirely based on the attenuation experienced by point correspondences accross multiple frames. We verify our algorithm with both synthetic and real data, and then compare it with an existing technique. Results obtained show significant improvement in the fidelity of the restored images.
\end{abstract}

\section{INTRODUCTION}

Underwater exploration of the seafloor is used for various scientific reasons such as assessing the biological environment, ${ }^{1,2}$ archaeological analysis, ${ }^{3}$ and taking population census ${ }^{2}$ etc. Analysis is typically performed on video recorded from sledges that move along the seabed. ${ }^{2}$ The elements involved in image capture are shown in Figure 1. As can been seen from the example captured image, high intensity light sources are usually employed due to the poor visibility underwater. These light sources usually leave a distinctive footprint of their beam on the seafloor (see figure 1). Within this footprint the illumination remains relatively constant, but beyond its boundary, it fades away. Multiple factors impact on the severity of this phenomenon, such as the illumination distribution of the light source, the natural vignetting due to the camera lens, and the radiometric response function of the camera. These components are shown in figure 1.

This non-uniformity of the brightness, as shown in figure 1, makes analysis of the seafloor (even manual inspection) difficult since the useful field of view is restricted. In this paper we propose a technique for improving the brightness of the scene by correcting for the various light degradation contributions from the light source, lens and CCD. The goal is therefore to generate a video sequence which is much easier to use and facilitates further automated analysis e.g. seabed classification ${ }^{1}$ and marine life inventory. ${ }^{2}$ An example of the result of our technique is shown in Figure 1.

In detail, we estimate the lighting footprint and the vignetting functions, which describe the illumination distribution of a light source, using multiple video frames captured with the same exposure setting. Previous work has addressed vignetting in consumer applications, mainly due to the lens and CCD, but certainly not the light source itself. Here we address a much more extreme form of vignetting (in effect), and the key novelties are i) the use of a more general vignetting model that uses an elliptical radial fall-off function, which ii) does not restrict its center to the image center, and ii) is robust to variations in scene depth.

The rest of this paper is organized as follows: in Section 2, we present previous work in this general area. Afterwards, we derive our overall light degradation model in Section 3, followed by the details of our technique in Section 4. An evaluation of our alorithm using both synthetic and real data is then presented in Section 5, along with comparisons with a state of the art technique for vignetting removal. Lastly, we conclude with a disscussion in Section 6.

This work was supported by the Science Foundation Ireland PI Programme: SFI-PI 08/IN.1/I2112 


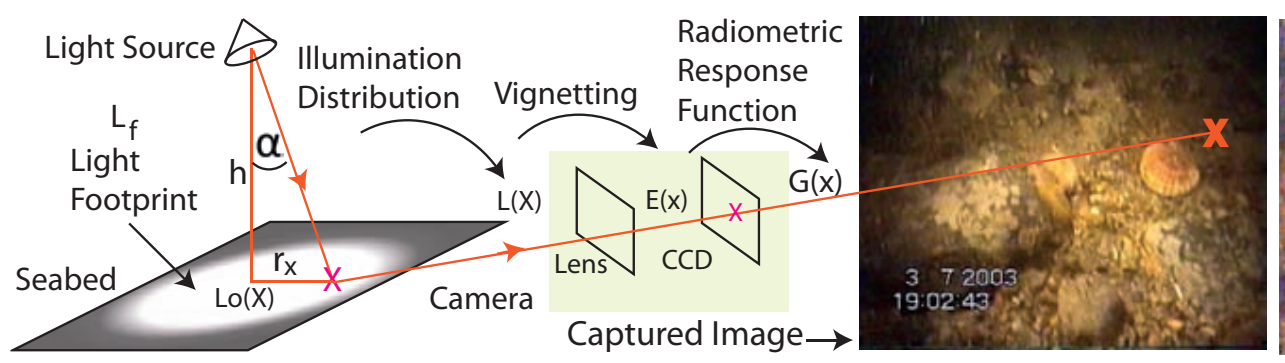

(a)

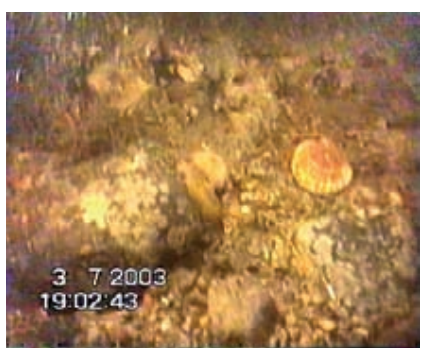

(b)

Figure 1. (a) Image formation process. The light source creates the scene radiance $L(\mathbf{X})$, which the vignetting from the camera lens transforms to image irradiance $E(\mathbf{x})$, which is then transformed into image brightness $I(\mathbf{x})$, by the camera response function $f()$, (b) Corrected Image

\section{PREVIOUS WORK}

There are two bodies of relevant literature. The first body of work improves underwater visibility based on physical models of light propogation underwater. Hence the colour degradation of an object underwater is modelled as a function of its distance from the camera, $z$. These methods usually require special equipment and/or user input. For example, in order to estimate lighting model parameters, Schechner and Korpel ${ }^{3}$ employed a special polarizer lens together with user selected areas in the image where there was only background. Sedlazeck et al. ${ }^{4}$ used user identified objects (limestone) and depth estimates to model colour degradation from a-priori knowledge of object colour. In our case the objects are at approximately the same depth, and we seek an automated solution to the problem.

The other body of work addresses the problem of vignetting directly, but not in our underwater case and certainly not for the extreme types of lighting degradations as we observe. These techniques employ models of the distortion function that is made up of a vignetting function and the camera response. The vignetting is typically assumed to be continuous, circular in shape, centered at the image centre, and is approximately the same in all frames. The parameters for these models can be estimated using either single or multiple images. Zheng et al. ${ }^{5}$ utilized the gradient along the radial direction from a single image for their vignetting parameter estimation, whereas the camera response, was assumed to be known. Kim et al. ${ }^{6}$ developed a robust method to estimate the vignetting and response functions independently and linearly based on the attenuations experienced among point correspondences between image pairs. In this method, the attenuations experienced at different radii were used to recover the vignetting, whereas for the response, attenuations at the same radii, taken at different exposures, were utilized. We have incorporated this methodology of using attenuations among point correspondences for estimating our vignetting function, because of the inherent independence to texture in the technique. In addition, we limit the vignetting correction to the region outside of the lighting footprint, as within this region the visibility of the image is good.

\section{DEGRADATION MODEL}

As shown in figure 1, the overall brightness of the captured scene is degraded along three stages of the image formation process, on the seabed, the camera lens and CCD. In the first stage, the radial fall off, $M_{L}(\mathbf{x})$, in the scene radiance, $L(\mathbf{x})$, can be primarly attributed to the light source illumination distribution on the seabed. Secondly, as $L(\mathbf{x})$ passes through the camera lens, it undergoes further degradation, which is commonly referred to as vignetting, $M_{o}(\mathbf{x})$. It has been shown in ${ }^{7}$ and, ${ }^{8}$ that these reductions in $L(\mathbf{x})$, can be modeled as $M_{L}(\mathbf{x})=\cos ^{3} \alpha_{L}(\mathbf{x})$ and $M_{o}(\mathbf{x})=\cos ^{4} \alpha_{o}(\mathbf{x})$. Where $\alpha_{L}(\mathbf{x})$ and $\alpha_{o}(\mathbf{x})$ are the angles between the normal to the surface at the center of the respective degradation function, and the point $\mathbf{x}$ under consideration. Then in the final stage of the image formation process, the captured $L(\mathbf{x})$ on the image plane, commonly reffered to as irradiance, $E(\mathbf{x})$, undergoes further adjustments in intensity and range by the camera exposure setting, $k$, and radiometric response function, $f()$. These adjustments result in a nonlinear mapping between $E(\mathbf{x})$ and the recorded image intensity, $G(\mathbf{x})$, which can be mathematically modelled together with $M_{L}(\mathbf{x})$ and $M_{o}(\mathbf{x})$ as:

$$
G(\mathbf{x})=f(k E(\mathbf{x}))=f\left(k L(\mathbf{x}) M_{L}(\mathbf{x}) M_{o}(\mathbf{x})\right)=f\left(k L(\mathbf{x}) \cos ^{3} \alpha_{L}(\mathbf{x}) \cos ^{4} \alpha_{o}(\mathbf{x})\right)
$$


Given the fact that we are interested in the overall effect of light attenuation through the system and not all of the image formation details, we have derived an effective degradation model, $B(\mathbf{x})$ as follows: Firstly, we generalize $f()$ as a gamma function, $\gamma$. Secondly, we assume $\alpha_{L}(\mathbf{x})=\alpha_{o}(\mathbf{x})=\alpha(\mathbf{x})$, which is resonable as $M_{L}(\mathbf{x})$ is the dominant attenuation in this case. Next, the ratio of $G(\mathbf{x}) / I(\mathbf{x})$, where $I(\mathbf{x})=f(k L(\mathbf{x}))$, is the notional unattenuated intensity of the image feature, is then taken to give:

$$
G(\mathbf{x}) / I(\mathbf{x})=B(\mathbf{x})=\left(k L(\mathbf{x}) \cos ^{3} \alpha(\mathbf{x}) \cos ^{4} \alpha(\mathbf{x})\right)^{\gamma} /(k L(\mathbf{x}))^{\gamma}=\cos ^{7 \gamma} \alpha(\mathbf{x})
$$

Afterwards, the expression $\cos \alpha(\mathbf{x})=h / \sqrt{r(\mathbf{x})^{2}+h^{2}}$, obtained from figure 1, is substituted into eq. 2, along with taking natural logarithms on either side to yield:

$$
\ln (G(\mathbf{x}) / I(\mathbf{x}))=\ln \left(\frac{h}{\sqrt{r(\mathbf{x})^{2}+h^{2}}}\right)^{7 \gamma}=\ln \left(\frac{1}{\sqrt{1+(r(\mathbf{x}) / h)^{2}}}\right)^{7 \gamma}=-\frac{7 \gamma}{2} \ln \left(1+(r(\mathbf{x}) / h)^{2}\right)
$$

where $r(\mathbf{x})=[\mathbf{x}-\mathbf{c}]^{T} \mathbf{V}[\mathbf{x}-\mathbf{c}]$ is the radius from image point $\mathbf{x}$ on the contour of an ellipse to its $\mathbf{c}=\left[c_{x}, c_{y}\right]^{T}$, with covariance matrix $\mathbf{V}=\left[v_{1}, v_{2} ; v_{2}, v_{3}\right]$ capturing the shape of the respective isophotes. In the next step of this simplification process, we substitute the Taylor series expansion of $\ln (1+x)=x+\sum_{n=2}^{\infty} a_{n}\left(x^{n}\right)$ for $|x| \ll 1$ as:

$$
\ln (G(\mathbf{x}) / I(\mathbf{x}))=\frac{-7 \gamma}{2} \ln \left(1+(r(\mathbf{x}) / h)^{2}\right)=\frac{-7 \gamma(r(\mathbf{x}) / h)^{2}}{2}+\sum_{n=2}^{\infty} a_{n}\left((r(\mathbf{x}) / h)^{2}\right)^{n}
$$

Then ignoring higher order terms by assuming $|r(\mathbf{x})| \ll 1$, as is the case due to the elements of $V$ are all being typically very small (i.e. $a b s\left(v_{1}, v_{2}, v_{3}\right) \ll 1$ ). This assumption was enforced by normalizing $r(\mathbf{x}), r_{n}(\mathbf{x})$, with the image dimentions i.e. $r_{n}(\mathbf{x})=r(\mathbf{x}) / \sqrt{w_{i d t h^{2}+h^{2} i g h t^{2}}}$. With this approximation, and incorporating $\gamma$ and $h$ into $r_{x}$, (i.e. $r^{\prime}(\mathbf{x})=r_{n}(\mathbf{x}) \sqrt{\gamma} / \mathrm{h}$ ), the effective degradation function, $B(\mathbf{x})$ can be expressed as:

$$
G(\mathbf{x}) / I(\mathbf{x})=B(\mathbf{x}) \approx \exp -\left(7 r^{\prime}(\mathbf{x})^{2} / 2\right)
$$

Note the following uniqueness of our degradation model compared to those utilized in the literature ${ }^{6},{ }^{3},{ }^{4}$. Firstly, we do not estimate $k$, but instead leave the images at the respective exposure setting utilized, as we assume this remained constant throughout the sequence. Secondly, $h$ was assumed to be aproximately constant over several frames, and hence there was no need its estimation nor $\gamma$, as they were both accounted for by the V parameters.

\section{UNDERWATER VIGNETTING CORRECTION}

Our goal is to estimate the parameters $\mathbf{c}$ and $\mathbf{V}$, so as to ultimately recover the original intensity $I(\mathbf{x})$. To do this we decouple the parameter estimation problem from the correction process in eq.5, and take a ratio of intensities of point correspondences, $A(\mathbf{x})$ in consecutive frames $G_{1}, G_{2}$ to yield

$$
\ln (A(\mathbf{x}))=\ln \left(\frac{G_{2}(\mathbf{x})}{G_{1}(\mathbf{x}+\mathbf{w}(\mathbf{x}))}\right)=\frac{-7}{2}\left(r_{2}^{2}(\mathbf{x})-r_{1}^{2}(\mathbf{x}+\mathbf{w}(\mathbf{x}))\right)
$$

where $r_{1}(\mathbf{x}+\mathbf{w}(\mathbf{x})), r_{2}(\mathbf{x})$, and $\mathbf{w}(\mathbf{x})$ are the corresponding positions and motion flow of image point $\mathbf{x}$ in frames $G_{1}, G_{2}$ respectively. Parameters $\mathbf{c}$ and $\mathbf{V}$ are then estimated in a Bayesian fashion. Hence the major steps, of our algorithm, in sequential order are: obtaining point correspondences, parameter estimation and correction.

\subsection{Correspondence}

Because of the inevitable inaccurances associated with the poor visibility conditions experienced in the underwater domain, as outlined in section 1, the full set of correspondences, based on the global motion flow, $\mathbf{w}(\mathbf{x})$ (i.e. $G_{2}(\mathbf{x})=G_{1}(\mathbf{x}+\mathbf{w}(\mathbf{x}))$ ), was initially obtained. The technique developed by Spindler and Bouthemy, ${ }^{9}$ for obtaining the dominant $2 \mathrm{D}$ motion from underwater video sequences with respect to the sea-bottom area, was employed for estimating $\mathbf{w}(\mathbf{x})$, where the affine motion model was utilized. 
Once the full set of corresponding points was obtained, we now selected a robust set for use in our vignetting estimation. This pre-selection process was based on the following criteria: intensity, edges, motion vectors, and attenuations values respectively. Underexposed and saturated points were removed by limiting the intensity range (25 to 235). Only points that had undergone significant motion ( $>2$ pixels), and attenuation $(|1-A(\mathbf{x})|>0.03)$, were selected. Lastly, to minimize the errors introduced as a result of the interpolating $G_{1}(\mathbf{x}+\mathbf{w}(\mathbf{x}))$ values for fractional values of $\mathbf{w}(\mathbf{x})$, points that were located on edges, of either frame, were not used. A canny edge detector was used for obtaining the respective edges.

\subsection{Parameter Estimation}

There are two sets of parameters to be estimated that would then yield intensity correction i) the center, $\mathbf{c}$ of the light distribution, and ii) shape matrix, $\mathbf{V}$ describing the shape of the light footprint in some sense. Proceeding in a Bayesian fashion, we require to maximise $p\left(\mathbf{c}, \mathbf{V} \mid G_{1}, G_{2}\right)$ w.r.t the paremeters $\theta=\left[c_{x}, c_{y}, v_{1}, v_{2}, v_{3}\right]$. Hence

$$
p\left(\theta \mid G_{1}, G_{2}\right) \propto p_{l}\left(G_{1}, G_{2} \mid \theta\right) p_{\theta}(\theta)
$$

The likelihood $p_{l}(\cdot)$ is derived directly from eq. $(6)$, while gaussian priors, $p_{\theta}(\cdot)$ were used for each parameter as:

$$
p_{l}\left(G_{1}, G_{2} \mid \theta\right) \propto \exp -\left[\frac{\left(\ln \left(G_{2} / G_{1}\right)+3.5\left({r^{\prime}}_{2}^{2}-r^{\prime 2}\right)\right)^{2}}{2 \sigma_{e}^{2}}\right] ; \quad p_{\theta}(\theta) \propto \exp -\left[\frac{\sum_{\mathbf{x}}\left(\theta-\theta_{0}\right)^{2}}{2 \sigma_{\theta}^{2}}\right]
$$

The parameters $\theta_{0}$ were estimated based on the predominantly illuminated region, $A_{m}(\mathbf{x})=\{0,1\}$, in the respective frame. We assume within this region corresponding points would experience the least (i.e. below average, $\left.\mu_{a}\right)$ attenuations, and thus was roughly estimated as $A_{m}(\mathbf{x})=|1-A(\mathbf{x})|<\mu_{a}$. Hence $\theta_{0}$ were were computed as:

$$
\mathbf{c}_{\mathbf{0}}=\frac{\sum_{\mathbf{x}} \mathbf{x} A_{m}(\mathbf{x})}{\sum_{\mathbf{x}} A_{m}(\mathbf{x})} ; \quad \mathbf{V}_{\mathbf{0}}^{-\mathbf{1}}=\frac{\sum_{\mathbf{x}}\left(\mathbf{x}-\mathbf{c}_{\mathbf{0}}\right)\left(\mathbf{x}-\mathbf{c}_{\mathbf{0}}\right)^{T} A_{m}(\mathbf{x})}{\sum_{\mathbf{x}} A_{m}(\mathbf{x})}
$$

The actual values for $\mathbf{c}$ and $\mathbf{V}$, were assumed not to be vastly different from these initial estimates, hence the individual components of $\sigma_{\theta}^{2}$ were set as follows: For the center, $\sigma_{x}^{2}$ and $\sigma_{y}^{2}$ were set to $25 \%$ the respective image width and height. While for the shape, $\sigma_{v 1}^{2}, \sigma_{v 2}^{2}$, and $\sigma_{v 3}^{2}$, were all set to $50 \%$ of their corresponding $\theta_{0}$ value. Then lastly, $\sigma_{e}^{2}$ was set to the actual variance obtained from the set of corresponding $\ln (A(\mathbf{x}))$ points.

\subsubsection{Optimisation Strategy}

Given the functions of likelihood and prior, the posterior expression for $p\left(\mathbf{c}, \mathbf{V} \mid G_{1}, G_{2}\right)$ in equation 7 is non-linear in the parameters. To simplify the process of maximization we choose to estimate the variables alternately using the well known Besag Iterated Conditional Modes (ICM) algorithm. ${ }^{10}$ Hence, we estimate $\mathbf{V}$ by maximising $p\left(\mathbf{V} \mid \mathbf{G}_{\mathbf{1}}, \mathbf{G}_{\mathbf{2}}, \mathbf{c}\right)$, holding $\mathbf{c}$ at its current estimate, then estimate $\mathbf{c}$ by maximisation of $p\left(\mathbf{c} \mid \mathbf{G}_{\mathbf{1}}, \mathbf{G}_{\mathbf{2}}, \mathbf{V}\right)$, holding $\mathbf{V}$ at its current estimate. This process was accomplished in three main steps. Firstly, we obtain the conditionals, which after examination of the posterior, eq. 8, also took Gaussian forms as follows:

$$
p\left(\mathbf{c} \mid G_{1}, G_{2}, \mathbf{V}\right) \propto p_{l}\left(G_{1}, G_{2}, \mid \mathbf{c}, \mathbf{V}\right) p_{\mathbf{c}}(\mathbf{c}) ; \quad p\left(\mathbf{V} \mid G_{1}, G_{2}, \mathbf{c}\right) \propto p_{l}\left(G_{1}, G_{2} \mid \mathbf{c}, \mathbf{V}\right) p_{\mathbf{v}}(\mathbf{V})
$$

These conditionals were then differentiated w.r.t. its relevant unknown, set to zero, and solved, using the robust method of Singular Value Decomposition (SVD). ${ }^{11}$ Then lastly, estimates for $\hat{\mathbf{c}}$ and $\hat{\mathbf{V}}$ were iteratively optimized as:

$$
\hat{\mathbf{c}^{n+1}}=\arg \max _{\mathbf{c}} p\left(\mathbf{c} \mid G_{1}, G_{2}, \mathbf{V}^{n}\right) ; \quad \mathbf{V}^{\hat{n}+1}=\arg \max _{\mathbf{v}} p\left(\mathbf{V} \mid G_{1}, G_{2}, \mathbf{c}^{n+1}\right)
$$

This optimization was performed until the percentage change between $(n+1)^{t h}$ and $n^{\text {th }}$ estimates for for each parameter in $\hat{\mathbf{c}}$ and $\hat{\mathbf{V}}$, was less than $5 \%$.

\subsection{Correction}

Given the parameters $\mathbf{V}$ and $\mathbf{c}$ estimated from the previous section, we can now correct the image. In practice however we find that it is more sensible to leave the already well-lit central region, $L_{f}$, untouched. Therefore we first estimate the extent of this region, and then boost the light intensity of the surrounding area. This practical procedure was achieved with the creation of a gain field, $C(\mathbf{x})$, which allowed us to control the apparent loss of contrast in the central area when performing correction. The estimation of $L_{f}$ and the creation of $C(\mathbf{x})$ and are illustrated in figure $2(\mathrm{~g})$ - (i), and are explained as follows: 


\subsubsection{Footprint Location:}

We estimate the extent of $L_{f}$ as the elliptical isophote radius, $r_{f}$, where a large percentage of image features had undergone a sudden significant (i.e. above average, $\mu_{a}$ ) increase in attenuation. This feature was identified as follows: Firstly, using the global attenuation field, $A(\mathbf{x})$ (eq. 6), all corresponding points which experienced above average attenuations, $A_{l}(\mathbf{x})$, was obtained:-

$$
A(\mathbf{x})=\frac{G_{2}(\mathbf{x})}{G_{1}(\mathbf{x}+\mathbf{w}(\mathbf{x}))} ; A_{l}(\mathbf{x})= \begin{cases}1 & |1-A(\mathbf{x})|>\mu_{a} \\ 0 & \text { otherwise }\end{cases}
$$

Then using the estimated parameters, $\mathbf{c}$ and $\mathbf{V}, A_{l}(\mathbf{x})$ was then segmented into a number $(y=20)$ of concentric bands, where $r_{f}$ was set as the boundary of the band where the largest percentage increase in $A_{l}$ points occured. These steps are illustrated in figure $2(\mathrm{~h})$.

\subsubsection{Gain Field Creation:}

A gain field, $C(\mathbf{x})$, was now created to correct the uneven illumination at each image location, $\mathbf{x}$, where within $r_{f}, C(\mathbf{x})$ was set to 1 , so as to leave this region untouched, whereas outside it was set to $1 / B(\mathbf{x})$, to correct the apparent vignetting effect. In practice however, there were a few image points, $d(\mathbf{x})$, outside $L_{f}$ that did not follow our degradation model $B(\mathbf{x})$, and were driven into saturation after $C(\mathbf{x})$ was initially applied. In most cases, these minor anomalies were due to suspended particles located much closer to the light source than the seafloor. To cater for this problem, $C(\mathbf{x})$ was set to 1 at these locations. Then lastly, to remove the discontinuity in the gain matrix at the footprint boundary, $C(\mathbf{x})$ was smoothed with a gaussian filter, $g_{\sigma}(\sigma=10)$, as shown in figure 2 (i). The creation steps just described, can be mathematically summarized, in numerical order, as:

$C(\mathbf{x})=\left\{\begin{array}{ll}1 & r(\mathbf{x}) \leq r_{f} \\ 1 / B(\mathbf{x}) & r(\mathbf{x})>r_{f}\end{array} ; d(\mathbf{x})=\left\{\begin{array}{ll}1 & G(\mathbf{x}) C(\mathbf{x})>255 \\ 0 & \text { otherwise }\end{array} ; C(\mathbf{x})=\left\{\begin{array}{ll}1 & d(\mathbf{x}) \\ C(\mathbf{x}) & \neq d(\mathbf{x})\end{array} ; C(\mathbf{x})=C(\mathbf{x}) * g_{\sigma} ;\right.\right.\right.$

With the gain field now created, the unattenuated image, $I(\mathbf{x})$, was now recovered as $I(\mathbf{x})=G(\mathbf{x}) C(\mathbf{x})$. However, throughout the survey, changes undergone in light source such as its intensity, depth, and orientation w.r.t. the seafloor alter the vignetting parameters. Hence, to correct all of the frames in the sequence, we re-estimated these parameters at each consecutive image pair. To cater for erronous global montion flow estimates, the change in these parameters were monitored over a 5 frame period, and hence was only updated if cummulative percentage difference was significant $(>5 \%)$, in at least 3 out of 5 consectutive frames.

\section{RESULTS}

In this section we evaluate the performance of our algorithm in estimating the shape, center and light footprint of various degradation functions, using synthetic and real data. The results obtained are also compared with the technique proposed by Kim et. al., ${ }^{6}$ noting that their work was directed at still and not moving images.

\subsection{Synthetic Experiments}

For our synthetic experiments, two 900 frame (235x380) sequences were created by translating multiple overlapping regions of a single image with known motion. For the first experiment, shown in figure 3, we examined the robustness of our algorithm with an elliptically shaped vignetting function, $V=[85,0 ; 0,45]$, centered at $\mathbf{c}=[165,107]$ (which was not the image center). The average Mean Absolute Error (MAE) of the degraded images was 84.7. Applying our algorithm, the recovered average values for the shape and center parameters were $V=[88.9,0 ; 0,48.1]$, and $\mathbf{c}=[171,103]$ (with standard deviations $\mathbf{V}_{\text {std }}=[4.9,0 ; 0,3.1]$, and $\mathbf{c}_{\text {std }}=[4,6]$ ), which yielded an average MAE of 9.7 after correction. These results show our algorithm can estimate elliptically shaped degradation functions that are not centered at the image center with a fair degree of accuracy. In comparison, Kim et. al. ${ }^{6}$ technique initially performed poorly giving an average MAE of 84.6 (as the vignetting was assumed to be circular and centered at the image center), but after our estimated radii parameters of $\mathbf{c}$ and $\mathbf{V}$ were incorporated into their process, good results were obtained, giving an average MAE of 10.3. This drastic improvement emphasises the need for good $\mathbf{c}$ and $\mathbf{V}$ estimates to perform vignetting removal efficiently. 


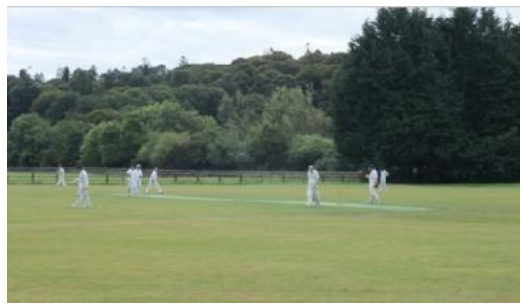

(a)

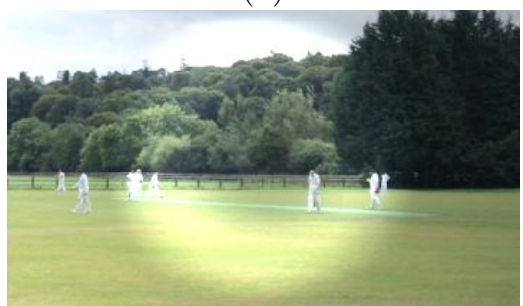

(d)

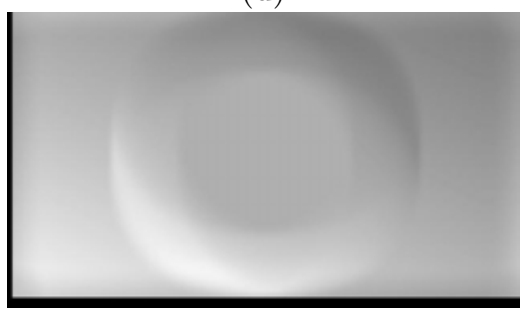

(g)

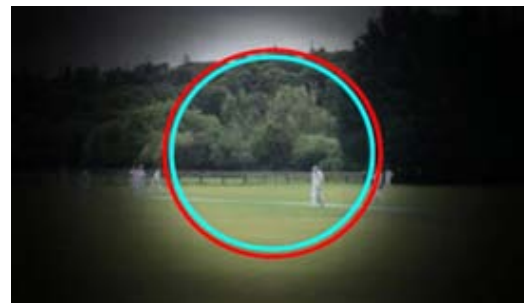

(b)

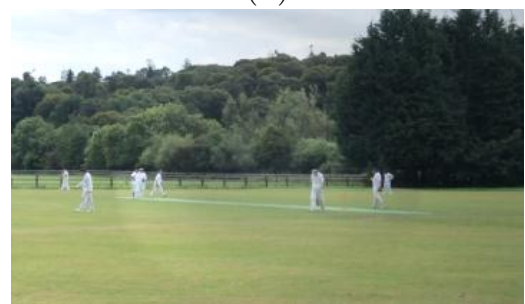

(e)

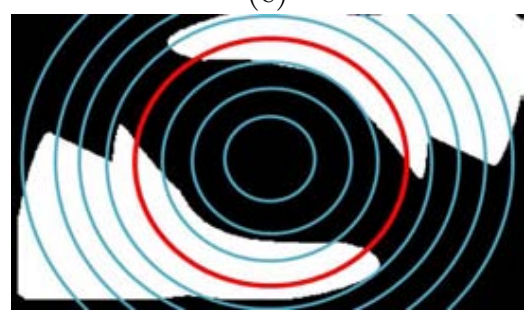

(h)

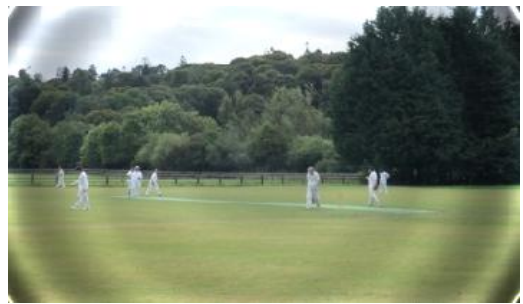

(c)

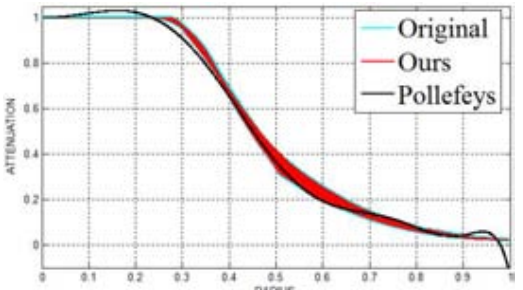

(f)

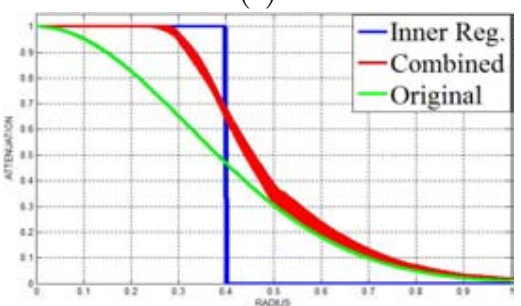

(i)

Figure 2. (a) Original (b) Artifically Vignetted, MSE=70.3, with simulated (blue) and recovered (red) footprint (c) Kim et. al. ${ }^{6}$ result, MSE $=10.6$ (d) Our result without footprint estimation, MSE $=25.1$ (e) Our Result with footprint estimation, $\mathrm{MAE}=8.7$ (f) Recovered degradation curves (g) Corresponding points intensity Ratios, $A(\mathbf{x})$ (h) Large $A(\mathbf{x})$ points, $A_{l}(\mathbf{x})$ (white), segmented into bands (blue), with footprint (red) identified where largest \% increase in $A_{l}(\mathbf{x})$ occured (i) Combining (via gaussian filtering) unity gain footprint (blue) region with degradation function (green) to create inverted gain field (red).

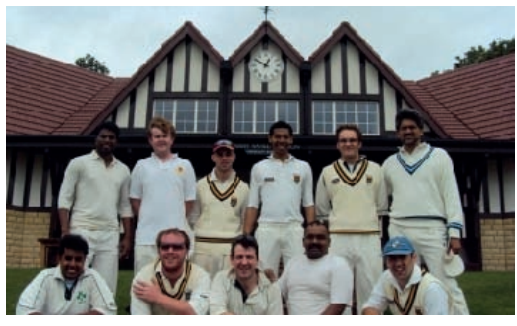

(a)

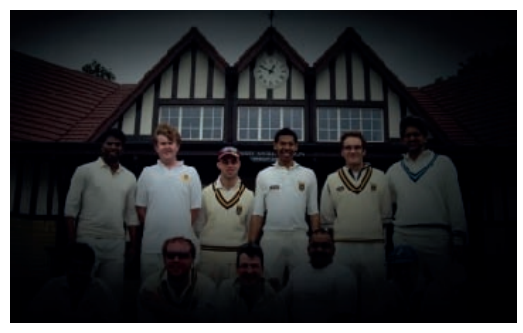

(b)

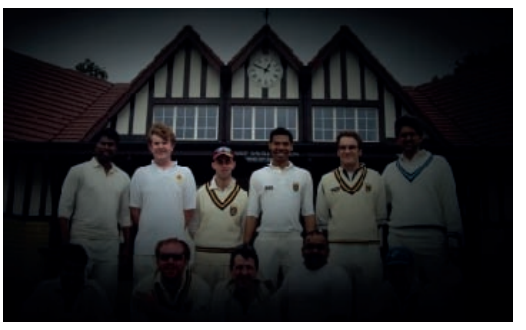

(c)

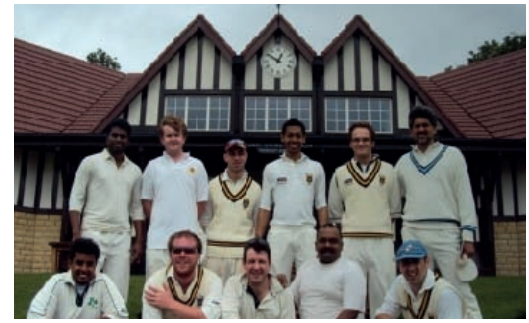

(d)

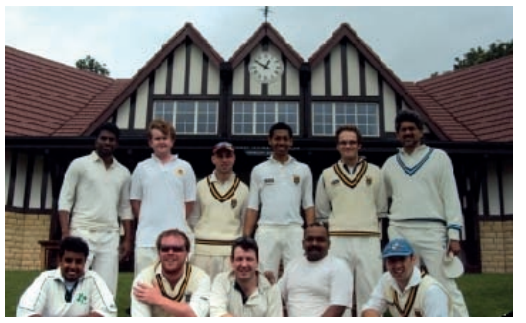

(e)

Figure 3. (a) Original (b) Artificially Vignetted, MSE $=84.7$ (c) Kim et al. ${ }^{6}$ Original Result, MSE=84.6 (d) Our Result, $\mathrm{MSE}=9.7$ (e) Result after incorporating our radii estimates of $\mathbf{c}$ and $\mathbf{V}$ into Kim et al. ${ }^{6}$ procedure, MSE $=10.3$ 
In our second experiment, shown in figure 2 , we degraded the images outside a central region, $r_{f}=0.4$, with a circular vignetting function, $V=[75,0 ; 0,75]$, centered at the image center, $\mathbf{c}=[190,117]$. The average MAE of the degraded images was 70.3. Applying our algorithm, we recovered average values for the shape, center, and footprint parameters of $V=[88.9,0 ; 0,48.1], \mathbf{c}=[171,103]$ and $r_{f}=0.3692$ (with standard deviations $\mathbf{V}_{s t d}=[5.1,0 ; 0,7.9], \mathbf{c}_{s t d}=[4,6]$, and $\left.r_{s t d}=0.05\right)$, which yielded an average MAE of 8.7 after correction. These results show our algorithm can estimate degradation functions which contain a centrally unattenuated region, with a fair degree of accuracy. Good results were also obtained from Kim et. al. ${ }^{6}$ (average MAE value of 10.6), the only issue being the wave-like appearance, as shown in figure 2 (c).

\subsection{Real Data}

For the real data, we used 400 frames from 5 sequences of video from real seabed surveys. Sample images of the results obtained are shown in figure 4 , and full sequences can be seen at www.sigmedia.tv/Misc/SPIE2012. As can be seen, our algorithm (with the footprint estimation incorporated), improved the lighting profile significantly, in comparison with Kim et. al., ${ }^{6}$ which initially performed poorly, but after our estimated radii parameters c and $\mathbf{V}$ were incorporated into their process, gave similar results to ours. It should be noted that in some cases, some image features were dirived into saturation after correction with Kim et. al. ${ }^{6}$ method, as seen in the last row in figure 2. These saturated regions were considered anomalies in our method, and were taken into account via our gain field creation step (see eq. 13), where their respective gains were set to unity.
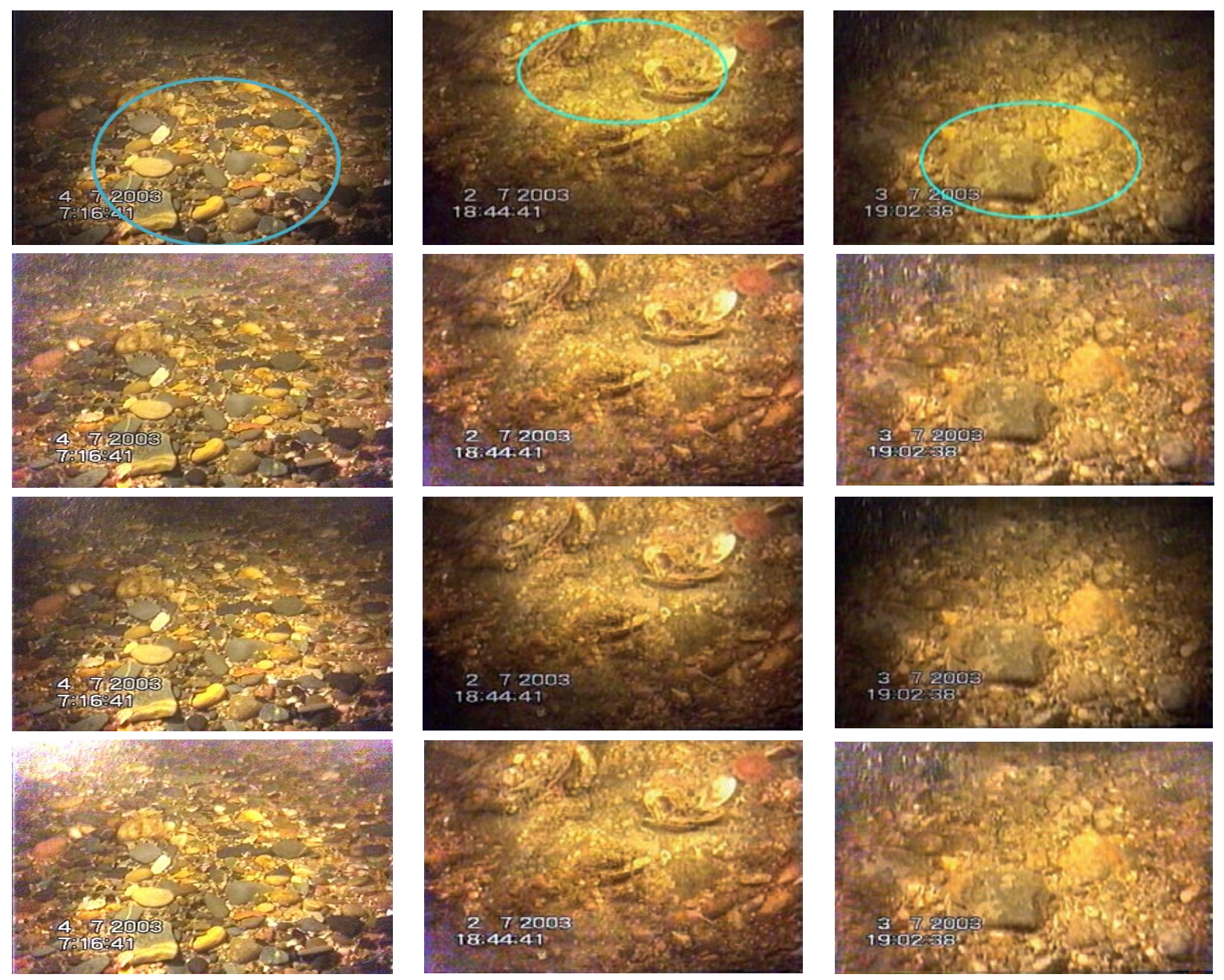

Figure 4. (Row 1) Original Degraded Images with estimated footprint superimposed in blue. (Row 2) Our Result. (Row 3) Kim et al. ${ }^{6}$ original result. (Row 4) Result after incorporating our radii estimates of $\mathbf{c}$ and $\mathbf{V}$ into Kim et al. ${ }^{6}$ procedure. 


\section{CONCLUSION}

This work has shown that it is possible to correct degraded video sequences from seabed surveys using ideas from the vignetting literature. We have introduced a new paramterisation of the problem and shown that it can achieve comparable/improved results to a well adopted model in the literature. The key ideas are firstly to estimate radii parameters, $\mathbf{V}$ and $\mathbf{c}$, based on the intensity ratios of point correspondences, and secondly to correct the distortion outside of the well lit region using an approximate gaussian function. The resulting sequences lighting profiles are improved significantly, which would aid in the seabed analysis, along with other visual applications such as mosaic creation etc.

In this paper, because of the uneven degradations in colours experienced in the underwater domain ${ }^{4}{ }^{3}$ the value channel from the HSV colour model was used to perform our estimation, which was subsequently corrected and then recombined with the original Hue and Saturation channels. In future work we would like to extend our method to deal with the attenuations experienced in each colour channel independently. Also, not to limit our degradation estimation to a gaussian shape, we would like to incorporate the flexibility of the polynomial vignetting model utilized in Kim et. al. ${ }^{6}$ into our correction process. However to avoid the wave-like effects encountered with this model, as seen in figure 2 (c), we would have to ensure its estimated function is monotonic.

\section{REFERENCES}

1. Lebart, K., Smith, C., Trucco, E., and Lane, D. M., "Automatic indexing of underwater survey video: algorithm and benchmarking method," Oceanic Engineering, IEEE Journal of 28(4), 673-686 (2003).

2. Campbell, N., D. H. and Bailey, N., "Investigating and mitigating uncertainties in the assessment of scottish nephrops norvegicus populations using simulated underwater television data," in [ICES Journal of Marine Science, 66], 646-655 (2009).

3. Schechner, Y. and Karpel, N., "Recovery of underwater visibility and structure by polarization analysis," Oceanic Engineering, IEEE Journal of 30(3), 570-587 (2005).

4. Sedlazeck, A., Koser, K., and Koch, R., "3d reconstruction based on underwater video from rov kiel 6000 considering underwater imaging conditions," in [OCEANS 2009-EUROPE, 2009. OCEANS '09.], 1-10 (2009).

5. Yuanjie, Z., Jingyi, Y., Sing Bing, K., Lin, S., and Kambhamettu, C., "Single-image vignetting correction using radial gradient symmetry," in [Computer Vision and Pattern Recognition, 2008. CVPR 2008. IEEE Conference on], 1-8 (2008).

6. Seon Joo, K. and Pollefeys, M., "Robust radiometric calibration and vignetting correction," Pattern Analysis and Machine Intelligence, IEEE Transactions on 30(4), 562-576 (2008).

7. of North America, I. E. S., [The IESNA Lighting Handbook], Illuminating Engineering Society of North America, New York, 9th ed. (2000).

8. Douglas A. Kerr, P., "Derivation of the cosine fourth law for falloff of illuminance across a camera image," 4, 2-5 (2007).

9. Spindler, F. and Bouthemy, P., "Real-time estimation of dominant motion in underwater video images for dynamic positioning," in [Robotics and Automation, 1998. Proceedings. 1998 IEEE International Conference on], 1063-1068 (1998).

10. Baesag, "On the statistical analysis of dirty pictures," Journal of the Royal Statistical Society. Series B (Methodological) Vol. 48(No. 3. (1986)), 259-30 (1986).

11. Wall, Michael E., A. R. L. M. R., "Singular value decomposition and principal component analysis," in $[A$ Practical Approach to Microarray Data Analysis], D.P. Berrar, W. Dubitzky, M. G., ed., 91-109, Kluwer: Norwell, MA (2003). 